\title{
Smectic Layering at the Free Surface of Liquid Crystals in the Nematic Phase: X-Ray Reflectivity
}

\section{Citation}

Pershan, Peter S., A. Braslau, A. H. Weiss, and J. Als-Nielsen. 1987. Smectic layering at the free surface of liquid crystals in the nematic phase: X-ray reflectivity. Physical Review A 35(11): 4800-4813.

\section{Published Version}

doi:10.1103/PhysRevA.35.4800

\section{Permanent link}

http://nrs.harvard.edu/urn-3:HUL.InstRepos:10357477

\section{Terms of Use}

This article was downloaded from Harvard University's DASH repository, and is made available under the terms and conditions applicable to Other Posted Material, as set forth at http:// nrs.harvard.edu/urn-3:HUL.InstRepos:dash.current.terms-of-use\#LAA

\section{Share Your Story}

The Harvard community has made this article openly available.

Please share how this access benefits you. Submit a story.

\section{Accessibility}




\title{
Smectic layering at the free surface of liquid crystals in the nematic phase: $\mathrm{X}$-ray reflectivity
}

\author{
P. S. Pershan, A. Braslau, and A. H. Weiss* \\ Division of Applied Sciences, Harvard University, Cambridge, Massachusetts 02138 \\ J. Als-Nielsen \\ Risø National Laboratory, Postbus 49, DK-4000 Roskilde, Denmark
}

(Received 19 September 1986)

\begin{abstract}
Smectic layering at the surface and smectic fluctuations in the bulk can be studied simultaneously by $x$-ray specular reflectivity and scattering measurements. There is a peak in the angular dependence of the specular reflectivity due to surface-induced smectic layering that penetrates into the bulk as $\exp \left(-z / \xi_{||}\right)$where $\xi_{||}$is one of the nematic critical correlation lengths. This is illustrated by measurements of the liquid-crystal materials octyloxycyanobiphenyl (8OCB) and butyloxybenzylidene octylaniline (4O.8). In both cases the critical divergence of the intensity of the peak in the specular reflectivity is weaker than $\xi_{\|}^{2}$, suggesting unexpected surface physics. The absolute value of the critical part of the density pair correlation function is determined by comparison to the Fresnel reflected intensity. Different geometries of the x-ray spectrometer are also discussed.
\end{abstract}

\section{INTRODUCTION}

Although $\mathrm{x}$-ray reflection from material surfaces is well known, there have been relatively few attempts to use the phenomena as a general technique for characterizing surfaces. ${ }^{1-8}$ In fact, classical techniques that have been employed at optical wavelengths are generally applicable in the $x$-ray range, with the advantage that the surface structure is probed on the scale of angstroms rather than microns. Current developments in the field of $\mathrm{x}$-ray optics testify to the accuracy of this assertion..$^{9-11}$ In this paper we describe a series of experiments carried out at the synchrotron facility HASYLAB (Hamburger Synchrotronstrahlungslabor, Deutsches Elektronen-Synchrotron) in Hamburg, Germany, on the x-ray reflectivity from the surface of the nematic phase of liquid crystals. ${ }^{12-14}$ The techniques developed for these experiments are generally applicable to any liquid surface. ${ }^{15,16}$ The principle physics addressed in this paper concerns the smectic-like order induced by a free surface of a nematic liquid crystal. The important feature of the nematic to smectic- $A$ phase transition is the critical divergence of the smectic susceptibility within the nematic phase. ${ }^{17}$ By simultaneously measuring the surface density profile and the critical scattering from the bulk, one can extract the absolute value of the critical fluctuations.

The two materials studied, octyloxycyanobiphenyl (8OCB) and butyloxybenzylidene (4O.8), have the phase sequence isotropic to nematic to smectic $A{ }^{18,19}$ The transition temperatures $\left(T_{I N}, T_{N A}\right)$ are approximately $\left(80^{\circ} \mathrm{C}, 67.1^{\circ} \mathrm{C}\right)$ for $8 \mathrm{OCB}$ and $\left(79^{\circ} \mathrm{C}, 63.5^{\circ} \mathrm{C}\right)$ for 40.8 . In both cases the isotropic-to-nematic transition is first order and the nematic-to-smectic- $A$ is second order; however, they differ in that for 40.8 the smectic layer spacing $d$ is essentially equal to the length of the molecule, while for $8 \mathrm{OCB}$, the molecules overlap each other to form bilayers. The layer spacing is approximately 1.6 times the length of the individual molecules. ${ }^{20}$
Classical treatment of the interaction between bound electrons and electromagnetic radiation at frequencies large compared to the electron binding energies obtains an x-ray dielectric constant $\epsilon=1-\rho r_{0} \lambda^{2} / \pi$, where $\rho$ is the electron density, $r_{0}$ the classical electron radius $e^{2} / m c^{2}$, and $\lambda$ the $x$-ray wavelength. ${ }^{21}$

Because $\epsilon<1$, $x$ rays incident on the surface at glancing angle $\phi_{i}$ will be refracted toward the surface at angle $\phi_{i}^{\prime}$. By Snell's law, $\cos \phi_{i}=\sqrt{\epsilon} \cos \phi_{i}^{\prime}$. Defining a critical angle $\phi_{c}$ by $\cos ^{2} \phi_{c} \equiv \epsilon$ and utilizing that the angles are small, i.e., $\phi_{c}^{2}=\lambda^{2} \rho r_{0} / \pi$, an approximate alternative form of Snell's law is $\phi_{i}^{2}=\phi_{i}^{\prime 2}+\phi_{c}^{2}$.

If $\phi_{i}$ is less than the critical angle $\phi_{c}, \phi_{i}^{\prime}$ is imaginary and, if one neglects scattering and absorption, the incident beam will be $100 \%$ reflected. If the discontinuity in the dielectric constant at the surface is sharp, the falloff in reflectivity with increasing $\phi_{i}$ can be calculated from the Fresnel formulas $R_{F}\left(\phi_{i}\right)$ of classical electromagnetic theory. Neglecting absorption, and for small $\phi_{i}$,

$$
\begin{aligned}
R_{F}\left(\phi_{i}\right) & =\left[\frac{\phi_{i}-\left(\phi_{i}^{2}-\phi_{c}^{2}\right)^{1 / 2}}{\phi_{i}+\left(\phi_{i}^{2}-\phi_{c}^{2}\right)^{1 / 2}}\right]^{2} \\
& =\left(\frac{\phi_{c}}{\phi_{i}+\left(\phi_{i}^{2}-\phi_{c}^{2}\right)^{1 / 2}}\right)^{4} .
\end{aligned}
$$

The effects of either a rough surface or a gradual surface profile will be discussed below. Nevertheless, whether the surface profile is sharp or not, an elementary property of classical optics is that for any flat surface the incident and reflected angles are equal to one another, and the reflected wave is in the plane defined by the incident wave vector and the surface normal. This is equivalent to the statement that if $\mathbf{k}_{i}$ and $\mathbf{k}_{s}$ are the incident and reflected wave vectors, respectively, then $\mathbf{Q}=\mathbf{k}_{s}-\mathbf{k}_{i}$ is normal to the surface. Alternatively, the scattering cross section for 
specular reflection from a flat surface (i.e., the $x-y$ plane) can be described as being proportional to a twodimensional $\delta$ function $\delta\left(Q_{x}\right) \delta\left(Q_{y}\right)$. This property of specular reflection allows it to be unambiguously separated from all other scattering processes.

The Fresnel reflectivity can be expressed in terms of the wave-vector transfer $Q \equiv 2 k \sin \phi_{i}$ and its critical value $Q_{c} \equiv 2 k \sin \phi_{c}$. For $Q \gg Q_{c}$, Eq. (1) becomes

$$
R_{F}(Q)=\left|\frac{Q_{c}^{2}}{4 Q^{2}}\right|^{2}
$$

For incident angles $\phi_{i}$ that are large compared to the critical angle $\phi_{c}$, the effects of a gradual surface profile can be understood in terms of the reflection coefficient for an electromagnetic wave incident on an infinitesimally thin slab of dielectric material, as given, for example, by Warren $^{21}$

$$
\frac{\delta E}{E_{0}}=4 \pi i r_{0}\left(\frac{\rho(z) d z}{Q}\right) \exp (i Q z),
$$

where $\rho(z) d z$ is the number of electrons per unit area in the slab of thickness $d z$ at height $z$. Since the refraction of $\mathrm{x}$ rays is negligible for $\phi_{i} \gg \phi_{c}$ and since the absorption is also not important for the materials and wavelengths of interest, the amplitude of the wave reflected from the graded surface is obtained by simply summing the amplitudes from successive thin layers. For a semi-infinite sample it is convenient to do one integration by parts to obtain

$$
\frac{E}{E_{0}}=-\left(\frac{4 \pi r_{0} \rho(\infty)}{Q^{2}}\right) \Phi(Q)=-\left(\frac{Q_{c}^{2}}{4 Q^{2}}\right) \Phi(Q),
$$

where $\rho(\infty)$ is the density far from interface and

$$
\Phi(Q)=\rho(\infty)^{-1} \int\left(\frac{d \rho}{d z}\right) \exp (i Q Z) d z .
$$

For an infinitely sharp surface, $\Phi(Q)$ is unity and Eqs. (3) and $\left(1^{\prime}\right)$ are consistent. The reflected intensity $R\left(\phi_{i}\right)$ is thus related to the reflectivity predicted by the Fresnel theory $R_{F}(Q)$ by

$$
\frac{R(Q)}{R_{F}(Q)}=|\Phi(Q)|^{2}, \quad Q \gg Q_{c} .
$$

In the special case that the derivative of the surface density is a Gaussian, e.g.,

$$
\begin{aligned}
& \rho(\infty)^{-1} \frac{d \rho}{d z}=\left(\sigma^{2} / 2 \pi\right)^{1 / 2} \exp \left(-\sigma^{2} z^{2} / 2\right), \\
& |\Phi(Q)|^{2}=\exp \left[\frac{-Q^{2}}{\sigma^{2}}\right) .
\end{aligned}
$$

If $Q_{c} / \sigma \ll 1$, Eq. (6) is applicable for all $Q$. For $Q<Q_{c}$, and in the absence of either scattering or absorption, the energy cannot propagate into the bulk of the material regardless of the value of $\sigma$. In this case, the reflectivity is essentially $100 \%$, independent of the density profile. For larger values of $Q$, structure, such as oscillations in the density profile due to smectic-like layers at the surface of a nematic liquid crystal, will result in a peak in $\Phi(Q)$, and thus a peak in the observed reflectivity, for $Q \approx 2 \pi / d$, where $d$ is the smectic layer spacing.

\section{SPECTROMETER CONFIGURATIONS}

The schematic of an $x$-ray spectrometer that is capable of scanning the wave-vector transfer component $Q_{z}$ perpendicular to the liquid surface is shown in Fig. 1(a). Two slits $S_{1}$ and $S_{3}$ are located at the same height, equal distances $L$ from the sample center. A scan in $Q_{z}$ is obtained by varying the incident angle $\phi_{i}$ and correspondingly the sample height $H_{s}=L \tan \phi_{i}$. We shall shortly describe in detail how the horizontal synchrotron beam can be bent downwards at any arbitrary angle $\phi_{i}$. Here it suffices to mention that the effect is obtained by the appropriate tilting of a monochromator crystal, cf. Fig. 2. At the same time, a monochromatic beam is extracted from the "white" synchrotron radiation spectrum. If the slit $S_{3}$ is not at the same height as $S_{1}$, an in-plane transverse wave-vector component $Q_{y}$ is selected. It is therefore possible by varying the incident angle, the sample height, and the slit height of $S_{3}$ to set the spectrometer for an arbitrary in-plane wave-vector transfer $\left(Q_{y}, Q_{z}\right)$ and still maintain a horizontal liquid surface as demanded by gravity. $S_{3}$ can also be moved horizontally, that is, perpendicular to the drawing plane of Fig. 1(a), and a general three-dimensional wave-vector transfer $\mathbf{Q}=\left(Q_{x}, Q_{y}, Q_{z}\right)$ is determined.

The resolution implied by finite slit openings is illustrated in Fig. 1(b). The central ray of incident and scattered wave vectors $\mathbf{k}_{i}$ and $\mathbf{k}_{s}$ is shown. The uncertainty $\delta \phi_{i}$ for the incident angle implies that the end point of possible incident wave vectors is distributed along the vector $\mathbf{X}_{i}$ with a certain characteristic width of $k \delta \phi_{i}$. Similarly, the end point of possible scattered wave vectors $\mathbf{k}_{s}$ are located along $\mathbf{X}_{s}$ and the actual wave-vector transfers are distributed within the shaded parallelogram spanned by $\mathbf{X}_{i}$ and $\mathbf{X}_{s}$. To be more precise, if the $\mathbf{k}_{i}$ and $\mathbf{k}_{s}$ distributions are boxlike, that, is constant along $\mathbf{X}_{i}$ and $\mathbf{X}_{s}$, respectively, and zero outside, then any wave-vector transfer to a point inside the parallelogram will be accepted by the spectrometer, while points that fall outside will be rejected. Alternatively, one might assume Gaussian distributions along $\mathbf{X}_{i}$ and $\mathbf{X}_{s}$ for $\mathbf{k}_{i}$ and $\mathbf{k}_{s}$. In that case, the spectrometer resolution function that describes the acceptance probability for various wave-vector transfers can be described by a two-dimensional Gaussian characterized by equal probability ellipses, where $\mathbf{X}_{i}$ and $\mathbf{X}_{s}$ are conjugate diameters. $^{22}$

The parallelogram in Fig. 1(b) indicates a long, thin resolution function corresponding to a situation where $\delta \phi_{s} \gg \delta \phi_{i}$, obtained by relaxing the slit height of $S_{3}$. Our experimental results showing perfect smectic layering at the surface imply that the corresponding cross section is a $\delta$ function in the transverse components $Q_{x}$ and $Q_{y}$. The effective $Q_{z}$ resolution for specular reflection is therefore not the total length of the parallelogram $\left(A A^{\prime}\right)$ but rather the section indicated by $\left(\delta_{z}\right)$ in Fig. 1(d).

In the bulk nematic phase, critical smectic fluctuations with correlation ranges $\xi_{\perp}$ and $\xi_{\|}$occur deep below the 
(a)

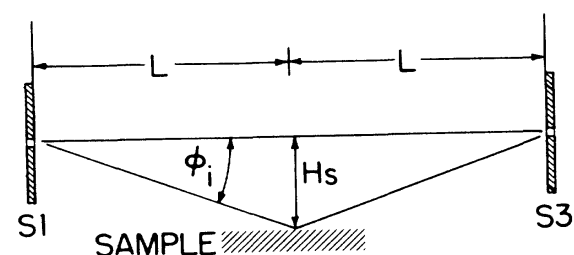

(b)

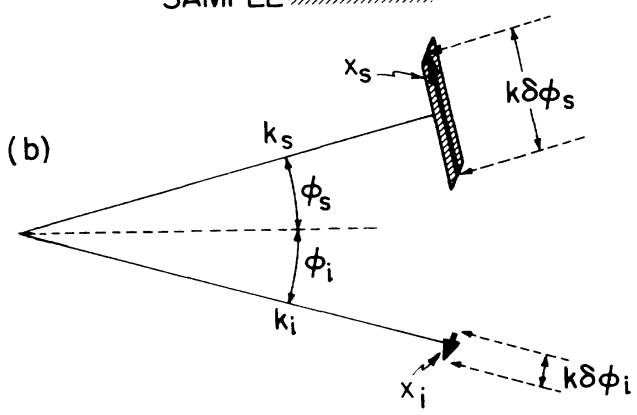

(c)

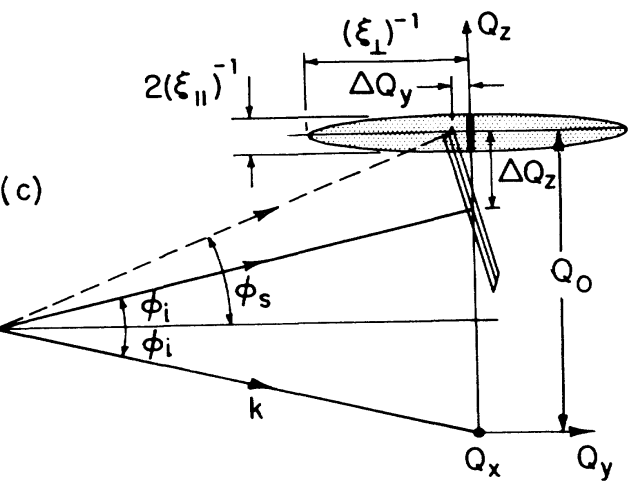

(d)

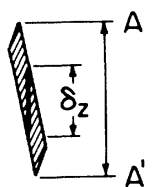

FIG. 1. (a) Schematic illustration of the spectrometer. When the incident angle $\phi_{i}$ is varied, the sample height $H_{s}$ is adjusted so $H_{s}=L \tan \phi_{i}$. With $S_{1}$ and $S_{3}$ at the same height, a $\phi_{i}$ scan is equivalent to a longitudinal scan with the wave-vector transfer normal to the liquid surface. (b) The angular resolution $\delta \phi_{i}$ and $\delta \phi_{s}$ for the incident and scattered beams $\mathbf{k}_{i}$ and $\mathbf{k}_{s}$, respectively, obtains the wave-vector resolution depicted by the two heavy lines $X_{i}=k \delta \phi_{i}$ and $X_{s}=k \delta \phi_{s}$. The convolution of the two obtains a resolution volume in reciprocal space with the cross-sectional area represented by the shaded parallelogram. (c) The kinematics of the lowresolution spectrometer. The shaded ellipse represents the half height contour for the critical scattering from the bulk nematic liquid crystal. The specular reflection is confined to the vertical $Q_{z}$ axis and the heavy line that is centered on the ellipse indicates the position in reciprocal space of the peak due to the surface layers. Even though the incident and scattered angles are not equal $\left(\phi_{i} \neq \phi_{s}\right)$, the finite resolution allows specular reflection. The spectrometer settings for this drawing correspond to the smallest value of $Q_{z}$ for which the critical bulk scattering can be detected. The center of the resolution volume is at $Q_{y}=0, Q_{z}=2 k \sin \left(\phi_{i}\right)=Q_{0}-\Delta Q_{z}$ and the end is at $Q_{y}=-\Delta Q_{y}, Q_{z}=Q_{0}$. (d) Detail of the resolution volume. The intersection of the resolution volume with the $Q_{z}$ axis, i.e., $\delta_{z}$, is the effective longitudinal resolution for specular reflection.

surface but still within the irradiated volume of the sample. The cross section for this bulk scattering is indicated in Fig. 1(c) by the elliptical, half-height contour ellipse with diameters $\xi_{\|}^{-1}$ and $\xi_{1}^{-1}$ along the $Q_{z}$ axis and $Q_{y}$ axis, respectively. This illustration is for the case when the spectrometer is set for $Q_{y}=0$. The intensity of bulk

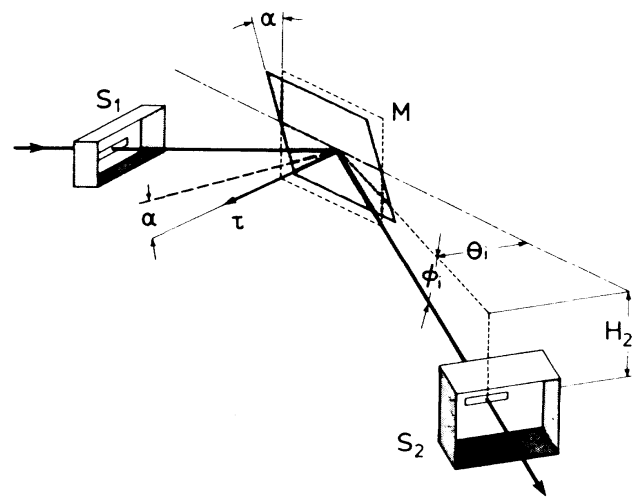

FIG. 2. The incident beam is bent downwards by the angle $\phi_{i}$ by tilting the monochromator $M$ by the amount $\alpha$. The "boxes" behind $S_{1}$ and $S_{2}$ indicate ionization chambers monitoring the beam intensity. scattering is then proportional to the overlap of the resolution function and the bulk cross-section ellipse.

The third wave-vector transfer dimension, perpendicular to the plane of the figures is readily included in the discussion. From this the following occurs.

(i) Relatively fine $Q_{z}$ resolution for specular reflection may be obtained even if the slit height of $S_{3}$ is relaxed so long as the illuminated portion of the surface is sufficiently flat.

(ii) The ratio of specular reflection to bulk scattering depends on the resolution and improves with narrow resolution.

(iii) The bulk scattering intensity may be determined separately by setting the spectrometer at a finite value of $Q_{y}$ (or $Q_{x}$ ) such that the signal for specular reflection at $Q_{x}=Q_{y}=0$ is outside of the resolution volume. By scanning $Q_{y}$ (or $Q_{x}$ ) at fixed $Q_{z}$, one may by extrapolation obtain the bulk intensity contribution at zero transverse wave-vector transfer and thereby separate surface from bulk scattering. An example is shown below in Fig. 14(b). Since the $Q_{y}$ resolution is always very high, one can also subtract off nonspecular reflection by scanning along $Q_{z}$ at a very small offset $Q_{y}$.

The bending of the "white" incident beam downward to any arbitrary glancing angle $\phi_{i}$ is illustrated in Fig. 2. The white, horizontal synchrotron beam is incident from the left on the slit $S_{1}$. The box behind $S_{1}$ indicates an 


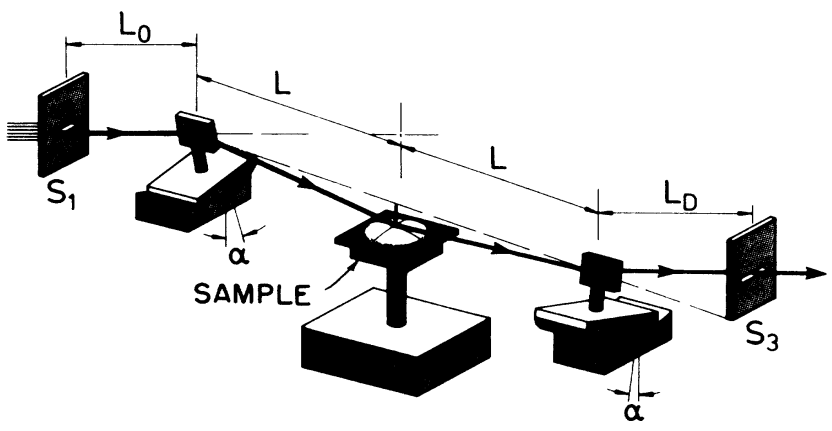

FIG. 3. A spectrometer configuration having a tilted analyzer crystal is used to obtain a resolution function in which the contributions from input and output sides are equal.

ionization chamber for monitoring the beam intensity after $S_{1}$. The beam is then Bragg reflected from a perfect $\mathrm{Si}$ or Ge crystal in the (111) or (220) orientation. The reciprocal-lattice vector $\tau$, normal to the reflecting planes and of magnitude $2 \pi / D, D$ being the lattice spacing, is at angle $\alpha$ with horizontal. The Bragg scattered beam is consequently bent downwards by an amount $\phi_{i} \simeq(\tau / k) \alpha$, where $k=2 \pi / \lambda$ is the magnitude of the wave vector. The Bragg angle is composed of the horizontal part $\theta_{i}$ and the vertical part $\phi_{i}$. In principle, this Bragg angle, and thereby the wavelength, varies if $\theta_{i}$ is kept fixed and $\phi_{i}$ is varied. Since $\theta_{i} \gg \phi_{i}$ in practice, the wavelength shift is very small and since the synchrotron spectrum is smooth, the corresponding intensity variation is negligible. This is monitored by the ionization chamber after a second slit $S_{2}$. In principle, $S_{2}$ can be wide open since the angle $\phi_{i}$ is completely determined by the tilt angle $\alpha$ and $\delta \phi_{i}$ is determined by the heights of $S_{1}$ and the electron beam 20 meters upstream. However, it is convenient to have a narrow slit $S_{2}$ just in front of the sample to provide a welldefined beam "footprint" on the sample surface and also because, in practice, the height setting of $\mathrm{H}_{2}$ gives a more accurate determination of $\phi_{i}$ than the goniometer setting $\alpha$. A slight error in $\alpha$ does not matter in that case because the counting time is determined by the $S_{2}$-monitor rate. In the measurements reported here, $S_{2}$ was left wide open.

The transverse, out-of-plane (i.e., horizontal) resolution is determined by the smaller of the two slit widths of $S_{1}$ and $S_{2}$, by the electron beam width (which are all of the order of $1 \mathrm{~mm}$ ), and by the large distance $(20 \mathrm{~m})$ from the slits to the source. If the direction of the detected beam is determined solely by the slit $S_{3}$ shown in Fig. 1, then the dominant contribution to the $Q_{x}$ resolution will be derived from the widths of the footprint on the sample surface together with the slit width of $S_{3}$ relative to the distance $L$ from sample to $S_{3}$, which is much shorter than the distance from sample to source. However, if a narrow $Q_{x}$ resolution is desirable, one can use a tilted analyzer crystal similar to the monochromator crystal, as shown in Fig. 3. ${ }^{13}$ The $Q_{x}$ resolution in this nondispersive geometry is then determined by the Darwin widths of the two perfect crystals. Furthermore, if the $Q_{x}$ resolution should not only be narrow but also have a very steep falloff (which may be useful in separating bulk scattering from surface scattering), one can use multibounce crystals. ${ }^{23}$ Here a groove is cut through a perfect crystal and three successive Bragg reflections take place, two from one face at the entrance and exit of the groove and one from the other face in the middle of the length of the groove.

Typical dimensions for both high resolution using an analyzer crystal and low resolution using a slit to define the scattered beam direction are given in Tables I and II.

\section{EXPERIMENTAL RESULTS}

\section{A. 8OCB high-resolution experimental results}

The surface reflectivity of octyloxycyanobiphenyl (8OCB) was measured using the high-resolution spectrometer described above. Figure 4 is a schematic drawing of the liquid-crystal oven. The samples were deposited on the surface of a glass flat, approximately $57 \mathrm{~mm}$ in diameter, that had been treated with a surfactant, $\mathrm{N}, \mathrm{N}$-dimethyl- $N$-octadecyl-3-aminopropyl-trimethoxysiyl chloride (DMOAP), in order to insure alignment of the long molecular axis normal to the glass surface. ${ }^{24}$ The amount of material $(1 \mathrm{~g})$ was chosen such that when heated into the isotropic phase it would form a broad flat drop, approximately $50 \mathrm{~mm}$ in diameter and $0.2 \mathrm{~mm}$ deep. By repeated slow cooling and heating through the nematic-to-smectic- $A$ transition it was possible to obtain a surface that is flat over most of the sample. The $\mathrm{x}$-ray reflectivity measurements to be described below are the best demonstration of this result.

The glass flat is mounted inside a sealed copper can with a glass window on top to view the sample and beryl-

TABLE I. Spectrometer parameters (high-resolution parameters). The distance, defined in Fig. 3, between the slit $S_{1}$ and the monochromator $L_{0}=140 \mathrm{~mm}$ for all configurations. In addition, for configurations 12.83 there was a slit $0.3 \mathrm{~mm}$ high by $20 \mathrm{~mm}$ wide, $3500 \mathrm{~mm}$ before slit $S_{1}$. ${ }^{3} \mathrm{Si}$ refers to a channel-cut crystal with three successive reflections. All slit dimensions are expressed as height times width.

\begin{tabular}{cccccccc}
\hline \hline & $\begin{array}{c}S_{1} \\
(\mathrm{~mm} \times \mathrm{mm})\end{array}$ & $\begin{array}{c}\text { Monochromator } \\
\text { and analyzer } \\
\text { crystals }\end{array}$ & $\begin{array}{c}\theta_{0} \\
(\text { degrees })\end{array}$ & $\begin{array}{c}\lambda \\
(\AA)\end{array}$ & $\begin{array}{c}L \\
(\mathrm{~mm})\end{array}$ & $\begin{array}{c}L_{D} \\
(\mathrm{~mm})\end{array}$ & $\begin{array}{c}S_{3} \\
(\mathrm{~mm} \times \mathrm{mm})\end{array}$ \\
\hline 6.82 & $0.02 \times 2$ & ${ }^{1} \mathrm{Ge}(111)$ & 13.642 & 1.540 & 575 & 600 & $1.0 \times 10$ \\
7.83 & $0.1 \times 0.7$ & ${ }^{3} \mathrm{Si}(111)$ & 14.221 & 1.537 & 545 & 545 & $0.1 \times 3.6^{\mathrm{a}}$ \\
\hline \hline
\end{tabular}

${ }^{\text {a }}$ Effective width due to width of channel in analyzer crystal. 
TABLE II. Spectrometer parameters (low- and intermediate-resolution parameters). See Table I for definitions.

\begin{tabular}{lcccccc}
\hline \hline Measurement & $\begin{array}{c}S_{1} \\
(\mathrm{~mm} \times \mathrm{mm})\end{array}$ & Monochromator & $\begin{array}{c}\theta_{0} \\
(\text { degrees })\end{array}$ & $\begin{array}{c}\lambda \\
(\AA)\end{array}$ & $\begin{array}{c}L \\
(\mathrm{~mm})\end{array}$ & $\begin{array}{c}S_{3} \\
(\mathrm{~mm} \times \mathrm{mm})\end{array}$ \\
\hline 8OCB 12.82 & $0.1 \times 0.7$ & ${ }^{1} \mathrm{Si}(111)$ & 14.211 & 1.536 & 575 & $7 \times 4$ \\
8OCB 3.83 & $0.1 \times 0.2$ & ${ }^{3} \mathrm{Si}(440)$ & 23.651 & 0.768 & 575 & $4 \times 0.2$ \\
& $0.1 \times 0.7$ & ${ }^{3} \mathrm{Si}(220)$ & 14.257 & 0.943 & 575 & $4 \times 0.2$ \\
& $0.1 \times 0.7$ & ${ }^{3} \mathrm{Si}(220)$ & 23.651 & 1.536 & 575 & $4 \times 0.2$ \\
4O.8 12.83 & $0.1 \times 0.7$ & ${ }^{3} \mathrm{Si}(111)$ & 14.221 & 1.537 & 620 \\
\hline
\end{tabular}

lium or Kapton $\mathrm{x}$-ray windows on the oven sides. Visible inspection of the isotropic-to-nematic phase transition, together with $\mathrm{x}$-ray measurements of critical phenomena, indicate that the sample temperature was homogeneous to better than a few millikelvin. The temperature of the oven was controlled by an electronic feedback circuit and varied by less than $10 \mathrm{mK}$ over periods of the order of hours.

The solid line in Fig. 5(a) is the x-ray reflectivity of an $8 \mathrm{OCB}$ sample at $50 \mathrm{mK}$ above the nematic-to-smectic- $A$ transition temperature $T_{N A}$ as a function of normalized wave vector $K=Q_{z} / Q_{0}$ measured with the highresolution spectrometer listed as " 6.82 " in Table II. The quantity $Q_{z}=(4 \pi / \lambda) \sin \phi_{i}$ and $Q_{0}=2 \pi / d$, where $d=31.6 \AA$, is the layer spacing in the smectic- $A$ phase of 8OCB. As mentioned above, with high resolution the only significant intensity to be detected is when the spectrometer is aligned exactly for specular reflection. Since the precision of the various goniometers is such that tilt of either the monochromator or analyzer was invariably accompanied by slight rotations compared to Darwin widths, each of these scans was taken by repeated optimization of the analyzer rotation and sample height for varying values of $Q_{z}$.

The dashed line in Fig. 5(a) is the Fresnel reflectivity $R_{F}(Q)$ for a flat surface with an abrupt discontinuity in the dielectric constant. The absolute value of the Fresnel reflectivity was measured to be greater than $90 \%$ below the critical angle $\phi_{c}$. In calculating $R_{F}(Q)$, absorption was included by considering a complex dielectric constant: The real part is given by

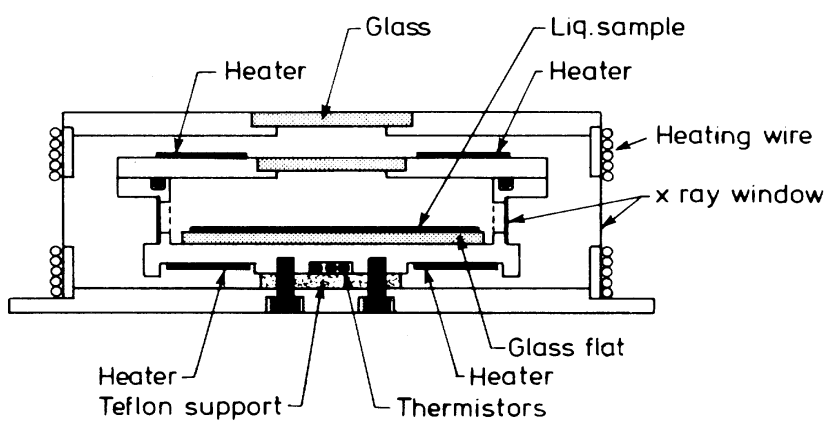

FIG. 4. Side view of the liquid-crystal oven used for reflectivity studies. The walls were made of copper. The sample covers a 2-in-diam area and the thickness is typically $0.2 \mathrm{~mm}$.

$$
\epsilon^{\prime}=1-\left(\frac{\rho \lambda^{2}}{\pi}\right)\left(\frac{e^{2}}{m c^{2}}\right)
$$

and the imaginary part $\epsilon^{\prime \prime}$ can be expressed in terms of the adsorption length $l$

$$
\epsilon^{\prime \prime}=\frac{\lambda}{2 \pi l} \text {. }
$$

For small $\phi_{i}=\phi$ the Fresnel reflectivity, Eq. (1), is then given by ${ }^{25}$

$$
R_{F}(\phi)=\frac{(\phi-\beta)^{2}+\left(\epsilon^{\prime \prime} / 2 \beta\right)^{2}}{(\phi+\beta)^{2}+\left(\epsilon^{\prime \prime} / 2 \beta\right)^{2}},
$$

where

$\beta^{2}=\frac{1}{2}\left\{\phi^{2}-\phi_{c}^{2}+\left[\left(\phi^{2}-\phi_{c}^{2}\right)^{2}+\left(\epsilon^{\prime \prime}\right)^{2}\right]^{1 / 2}\right\}$

for $\phi>\phi_{c}$

or

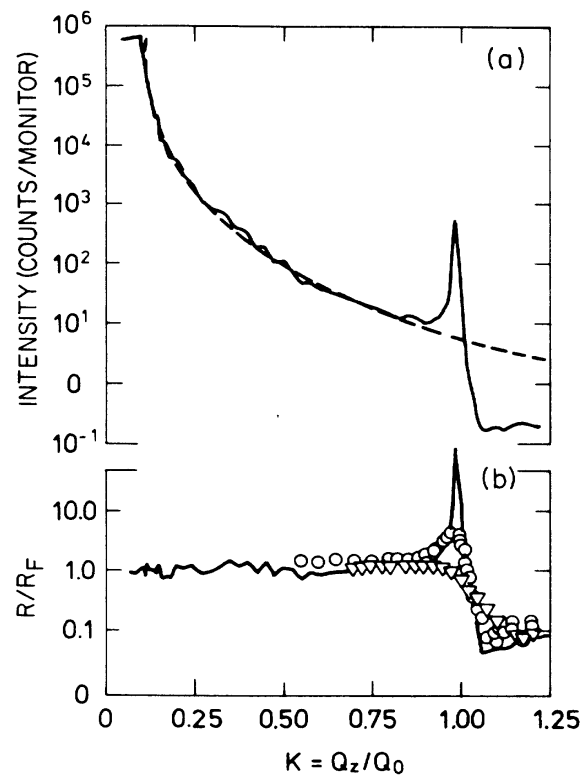

FIG. 5. (a) High-resolution longitudinal scan of the reflected intensity from the surface $8 \mathrm{OCB}$ at $T-T_{N A}=0.05^{\circ} \mathrm{C}$. The $Q_{z}$ scale is normalized to $Q_{0}=0.199 \AA^{-1}$. The dashed line is the calculated Fresnel reflection law. (b) Reflected intensity vs $Q_{z} / Q_{0}$ divided by the Fresnel law. The solid line for $T-T_{N A}=0.05^{\circ} \mathrm{C}$, open circles for $T-T_{N A}=2.8^{\circ} \mathrm{C}$, and triangles for $T-T_{N A}=11.06^{\circ} \mathrm{C}$. 
$\beta^{2}=\frac{1}{2} \frac{\left(\epsilon^{\prime \prime}\right)^{2}}{\phi_{c}^{2}-\phi^{2}+\left[\left(\epsilon^{\prime \prime}\right)^{2}+\left(\phi_{c}^{2}-\phi^{2}\right)^{2}\right]^{1 / 2}}$

$$
\text { for } \phi<\phi_{c} \text {. }
$$

The effects of absorption are only noticeable very near to the critical angle $\left(\phi \approx \phi_{c}\right)$. The solid line in Fig. 5(b) displays the result of dividing the measured reflectivity of Fig. 5(a) by $R_{F}$ using $\phi_{c}=0.152^{\circ}$ and $\epsilon^{\prime \prime}=1.16 \times 10^{-8}$. The open circles and the triangles display similar results for $T-T_{N A}=2.8$ and $11.06^{\circ} \mathrm{C}$, respectively.

The half width at half maximum of the temperaturedependent peak at $Q_{z}=Q_{0}$ or $K=1$ is found to be equal to $1 / \xi_{\|}(T)$, where $\xi_{\|}(T)$ is the longitudinal correlation length of the critical fluctuations as previously measured in bulk $8 \mathrm{OCB} .{ }^{18}$ The combination of this, together with the fact that the signal is only observed for the precise specular reflection conditions, shows that the peak is due to smectic layers parallel to the sample surface and penetrating into the bulk of the nematic phase, one bulk correlation length.

It is interesting to note that for $Q_{z}<0.8 Q_{0}$ the reflectivity is equal to the Fresnel value independent of temperature. Furthermore, for $Q_{z}>Q_{0}$, the reflectivity falls below the Fresnel value rather sharply, with only a slight temperature dependence. The reflectivity line shape, modeled by assuming the average of the electron density over the $x-y$ plane, can be written as the sum of two terms: $\rho(z)=\rho_{0}(z)+\rho_{1}(z)$. The intuitively obvious form for the first term is taken to be

$$
\begin{aligned}
\frac{1}{\rho(\infty)} \frac{d \rho_{0}}{d z}= & \frac{\partial}{\partial z} \Theta\left(z-z_{0}\right) B_{s} \exp \left(-\frac{z-z_{0}}{\xi_{\|}(T)}\right) \\
& \times \sin \left[Q_{0}\left(z-z_{0}\right)\right],
\end{aligned}
$$

where

$$
\Theta\left(z-z_{0}\right)=\left\{\begin{array}{ll}
0, & \text { if } z<z_{0} \\
1, & \text { if } z>z_{0}
\end{array} .\right.
$$

A second term is necessary to describe the transition from the Fresnel reflectivity for $Q<Q_{0}$ to the lower reflectivities at $Q>Q_{0}$. A form which represents the data very well is

$$
\frac{1}{\rho(\infty)} \frac{d \rho_{1}}{d z}=C_{1}(\pi z)^{-1} \exp \left(-\frac{(\sigma z)^{2}}{2}\right) \sin Q_{1} z,
$$

where $C_{1}$ is defined to ensure $\int \rho^{-1} d \rho_{1} / d z=1$. It follows that

$$
\Phi(Q)=\Phi_{0}(Q)+\Phi_{1}(Q),
$$

where

$$
\begin{aligned}
\Phi_{0}(Q)=+ & i\left(\frac{B_{s}}{2}\right) \exp \left(-i Q z_{0}\right) \\
& \times\left(\frac{\xi_{\|} Q_{0}-1}{\left(Q_{z}-Q_{0}\right) \xi_{\|}-i}-\frac{\xi_{\|} Q_{0}+1}{\left(Q_{z}+Q_{0}\right) \xi_{\|}+i}\right)
\end{aligned}
$$

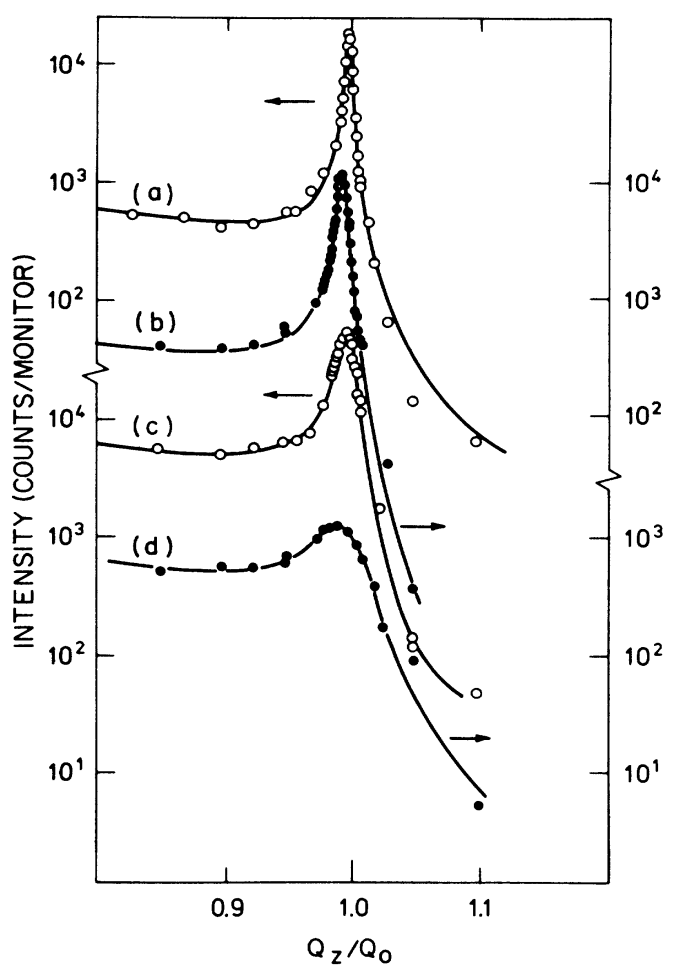

FIG. 6. High-resolution, specular reflectivity data for $8 \mathrm{OCB}$ near the peak due to the surface layers. The open circles refer to the left scale and the solid circles to the right scale. The solid lines are calculated from the model described in the text with the parameters in Table III. The temperatures $T-T_{N A}$ are (a) $0.10^{\circ} \mathrm{C}$, (b) $0.21{ }^{\circ} \mathrm{C}$, (c) $0.40^{\circ} \mathrm{C}$, and (d) $1.80^{\circ} \mathrm{C}$.

$$
\Phi_{1}(Q)=\left(\frac{C_{1}}{2}\right)\left[\operatorname{erf}\left(\frac{Q+Q_{1}}{\sqrt{2} \sigma}\right)-\operatorname{erf}\left(\frac{Q-Q_{1}}{\sqrt{2} \sigma}\right)\right]
$$

with $C_{1}^{-1}=\operatorname{erf}\left(Q_{1} / \sqrt{2} \sigma\right)$.

The solid lines in Fig. 6 represent the results of nonlinear least-square fits of the measured results for four different temperatures to the above functional form convoluted with a Gaussian resolution function. The full width at half maximum $\left(\Delta Q_{z}\right)$ of the resolution function was obtained by measuring the peak line shape near, but just below, $T_{N A}, \Delta Q_{z}=\left(1.7 \times 10^{-3}\right) Q_{0}$. The temperatures and other parameters in the fits are described in Table III.

TABLE III. Results of nonlinear least-squares fits to the high-resolution data on 8OCB. The parameter $z_{0}$ was fixed at $0.25 d$.

\begin{tabular}{cccccc}
\hline $\begin{array}{c}T-T_{N A} \\
\left({ }^{\circ} \mathrm{C}\right)\end{array}$ & $B_{s}$ & $\xi_{\|} Q_{0}$ & $Q_{0} / \sigma$ & $\chi^{2}$ & $Q_{1} / Q_{0}$ \\
\hline 0.10 & 0.043 & $450 \pm 30$ & $100 \pm 80$ & 10 & 1.001 \\
0.21 & 0.065 & $275 \pm 15$ & $18 \pm 7$ & 5 & 1.05 \\
0.40 & 0.067 & $145 \pm 7$ & $16 \pm 8$ & 7 & 1.04 \\
1.80 & 0.099 & $45.4 \pm 7$ & $7.7 \pm 1.8$ & 3 & 1.03 \\
\hline \hline
\end{tabular}


The best fit values of $\xi_{\|}(T)$ agree, within experimental error, with values previously published ${ }^{18}$ for the critical correlation length in bulk 8OCB. The value for $z_{0}$, the origin of the exponentially damped oscillations, was held fixed at $0.25 d$. In real space this value of $z_{0}$ locates the maxima in $\rho_{0}$ exactly $d / 2$ away from the surface as defined by the maxima in $d \rho_{1} / d z$. Nonlinear least-squares fits in which $z_{0}$ is allowed to vary freely converge around $z_{0} \approx 0.25 d$; however, the fact that the $B_{S}$ and $z_{0}$ are highly correlated, results in random variations that we do not believe to be meaningful. In fact, $z_{0} \approx 0.25 d$ is necessary to obtain asymmetries in the peak line shapes that are shown in Fig. 6. An example of a best fit keeping $z_{0}$ fixed at $0.5 d$ is shown in Fig. 7. Clearly $z_{0}=0.25 d$ is a better choice.

This particular choice for the representation of $\rho_{1}$ emerged as the simplest parametrization of a model which could reproduce both the agreement with the Fresnel law for $Q / Q_{0}<0.8$ and the falloff at larger $Q$. In particular, the simple Gaussian form that was used to represent either the structureless surface of water ${ }^{15}$ or the $\Phi_{1}$ term used to describe the surface of the isotopic phase of dodecylcyanobiphenyl (12CB) (Ref. 26) does not provide a good fit to the present data. In the absence of a more basic model, the above form has the advantage that it provides a quantitative representation of the fact that the exponentially decaying $\rho_{0}$ term described by Eq. (11) does not accurately describe the smectic oscillations near the surface. Furthermore, we believe that the form for $\rho_{1}$ given by $\mathrm{Eq}$. (12) does indeed provide an accurate representation of the quantative features of the physical surface.

In view of the fact that the falloff in $\Phi_{1}(Q)$ occurs in the vicinity of $Q \approx Q_{0} \approx Q_{1}$ its precise shape is partially obscured by the peak in $\Phi_{0}(Q)$. In particular, without precise measurements in the tails of $\Phi(Q)$, the best that

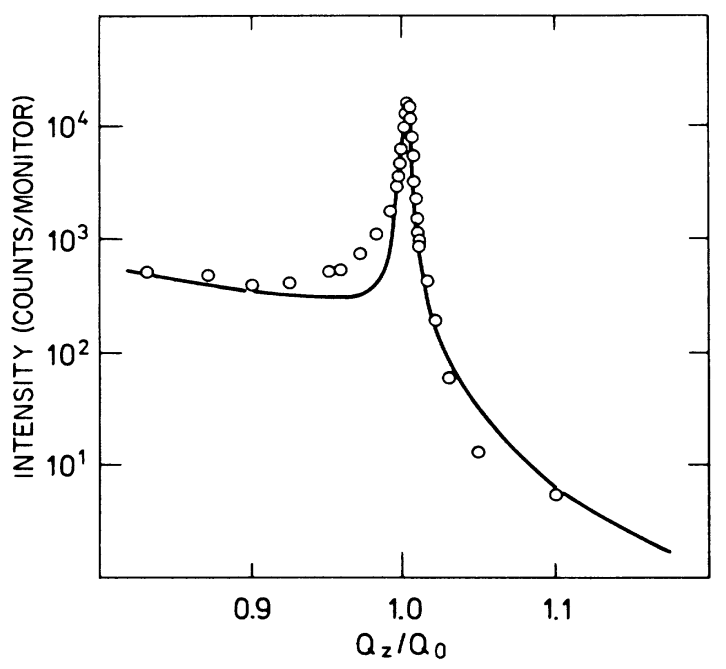

FIG. 7. Effect of varying the phase of the two terms that make up $\Phi(Q)$. The data are the 8OCB data in Fig. 6(a) but the solid line is the best fit, keeping $z_{0}$ fixed at a value of $0.5 \mathrm{~d}$. can be done is to set a lower limit to the Gaussian penetration length, $1 / \sigma$. Furthermore, where the peak is relatively intense, the numerical results for $B_{s}$ and $\xi_{\|}$are insensitive to the precise value of $\sigma$. Figure 8 displays the values of $Q_{0} / \sigma$ as obtained from fits to the data sets described in Table III. In addition, we also display results from other data sets that were less complete than the above (and thus not otherwise included here) but which were adequate to set lower limits on $Q_{0} / \sigma$. One can see from Fig. 8, that although the Gaussian penetration length $1 / \sigma$ is continually driven to larger and larger values as $T$ approaches $T_{N A}$, its precise value is rather uncertain. The solid line is the best fit to the exponential correlation length $\xi_{\|} Q_{0}$ as obtained from critical scattering from the bulk of $8 \mathrm{OCB} .{ }^{18}$ Since the falloff of $\rho_{1}$ in real space is dominated by the Gaussian form, while the falloff in $\rho_{0}$ is governed by the longer-range exponential, the density oscillations described by $\rho_{1}$ are considerably more localized at the surface than those described by $\rho_{0}$. Nevertheless, as $T$ approaches $T_{N A}$, the $\rho_{1}$ oscillations extend deeper and deeper into the sample.

The $\chi^{2}$ values reported in Table III were based on the assumption that the statistical weight for each point was given by Poisson statistics. The fact that the $\chi^{2}$ values for these fits are significantly larger than unity is a quantitative demonstration that either the empirical form is not strictly correct, or that there are other statistical uncertainties beyond the counting statistics. Both of these are likely to be true. Nevertheless, in view of the fact that very small systematic errors will make sizable contributions to data sets like this, which encompass large dynamic ranges, these values for $\chi^{2}$ are not unreasonable.

This particular model, while not necessarily unique, does illustrate our contention that the only way to obtain a functional form that will be flat out to $Q_{z} \sim Q_{0}$ and then fall off rather abruptly for $Q_{z}>Q_{0}$ is to include spatial

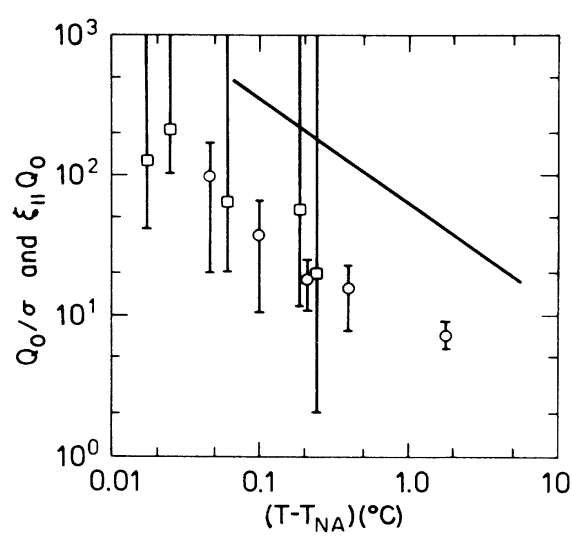

FIG. 8. The Gaussian penetration length $Q_{0} / \sigma$ for $8 \mathrm{OCB}$ as a function of $T-T_{N A}$. The open circles are the results of the fits to the data shown in Fig. 6. The open squares are from less complete data sets from which it was not possible to obtain upper error limits. The solid line represents the published values for $\xi_{\|} Q_{0}$ (Ref. 18). 
oscillations in the electron density that are localized to the vicinity of the surface. The model-independent physical significance of this is that the exponential decay included in the $\Phi_{0}(Q)$ term breaks down in the vicinity of the surface. Furthermore, since the surface structure responsible for the principle features of the model for $\Phi_{1}(Q)$ persist to much higher temperatures than the critical phenomena associated with the bulk, one cannot ignore the possibility of surface phases with separate critical properties from that of the bulk.

\section{B. 8OCB low resolution}

We shall now consider the situation when the resolution for the scattered radiation is relaxed by removing the analyzer crystal and using a slit $S_{3}$ with a rather open aperture $(7 \mathrm{~mm}$ high by $4 \mathrm{~mm}$ wide, see Table II, entry 12.82). First we shall give a qualitative discussion then present some data, and finally explain how the data are analyzed quantitatively.

Figure 1(c) shows the scattering diagram in reciprocal space. The heavy lines, downwards and upwards at angle $\phi_{i}$ represent the incoming and the specular reflected wave vectors, respectively. The high slit height $Y_{3}$ of slit $S_{3}$ at distance $L$ from the sample implies that the dashed outgoing wave vector at angle $\phi_{s}=\phi_{i}+\Delta \phi_{s}$ will also be detected, $\Delta \phi_{s} \approx Y_{3} / 2 L$. Since the scattering is elastic, this process has a wave-vector transfer component in the $Q_{y}$ direction, $\Delta Q_{y}$, as well as the $z$ component $\Delta Q_{z}$ added to the central ray. Inspection of Fig. 1(c) immediately leads to

$$
\begin{aligned}
& \frac{\Delta Q_{z}}{Q_{0}} \approx\left(\frac{k}{Q_{0}}\right) \Delta \phi_{s} \approx \frac{\Delta \phi_{s}}{2 \phi_{0}}, \\
& \frac{\Delta Q_{y}}{Q_{0}} \approx \phi_{i} \frac{\Delta Q_{z}}{Q_{0}} \approx \frac{\Delta \phi_{s}}{2},
\end{aligned}
$$

since $\phi_{0}$ is defined such that $2 k \phi_{0} \approx Q_{0}$.

The short side of the resolution parallelogram is given by the angular divergence $\delta \phi_{i}$ of the incident beam as $k \delta \phi_{i}$. The thickness of the resolution parallelogram along the $z$ axis is thus indicated in Fig. 1(d) by $\delta_{z} / Q_{0}$ $=2\left(k / Q_{0}\right) \delta \phi_{i} \approx \delta \phi_{i} / \phi_{0}$. The high effective resolution for specular reflection in this low-resolution geometry is quite remarkable. The selection rule that confines specular reflection to the $z$ axis, together with the small tilt $\phi_{0}$ of the long resolution parallelogram, results in a width $\delta_{z} / k$ that is only twice the width of the incident beam $\delta \phi_{i}$.

There are three contributions to the scattering cross section: simple specular reflection confined to the $z$ axis and decaying with increasing $Q_{z}$ roughly as $\left(Q_{c} / 2 Q_{z}\right)^{4}$, reflection from the smectic layering at the surface confined to the $z$ axis and peaking at $Q_{z}=Q_{0}$ with a half width of $\left(\xi_{||}\right)^{-1}$, and finally the scattering from critical smectic fluctuations in the bulk peaking at $Q_{z}=Q_{0}, Q_{y}=0$, with half widths $\left(\xi_{\|}\right)^{-1}$ and $\left(\xi_{\perp}\right)^{-1}$ along the $z$ and $x, y$ directions, respectively. It is evident from Fig. 1(c) that this latter contribution is expected to be roughly constant in a $Q_{z}$ scan for $\left|Q_{z}-Q_{0}\right|<\Delta Q_{z}$, provided that $\Delta Q_{y} \ll\left(\xi_{1}\right)^{-1}$, and rapidly vanishes outside this interval.

Experimental data obtained with the conditions listed in Table II at entry 12.82 are displayed in Fig. 9. From Table II one obtains the values of $\Delta Q_{z}=0.122 Q_{0}$, $\Delta Q_{y}=0.0030 Q_{0}$, and $\delta_{z}=0.004 Q_{0}$. The data in Fig. 9 were taken at $T-T_{N A}=0.164^{\circ} \mathrm{C}$. From either the published data for $\xi_{\|}(T)$ (Ref. 18) or the high-resolution scans like that in Fig. $6, \xi_{\|} Q_{0}=250$. Similarly, the published data obtain $\xi_{1} Q_{0}=29$. ${ }^{18}$ Thus we have the necessary condition for the critical scattering from the bulk to be constant, i.e., $\Delta Q_{y} \xi_{1}<<1$, and we see that the data in Fig. 9 have the expected flat plateau in the interval $\left|Q_{z} / Q_{0}-1\right|<\Delta Q_{z} / Q_{0}=0.122$. The peak due to the surface layers at $Q_{z} / Q_{0}=1$ also has the full width at half maximum of $2\left(\xi_{\|} Q_{0}\right)^{-1}=8 \times 10^{-3}$ that is expected when $\delta_{z} \ll 2 / \xi_{||}$. Finally, these two contributions are superimposed on a sloping "Fresnel background" as indicated by the dashed line calculated from Eq. (1).

For this spectrometer, as for the high-resolution spectrometer, the relative intensity between the surface peak at $Q_{z} / Q_{0}=1$ and the Fresnel background measures the amplitude $B_{s}$. However, now the critical scattering from the bulk obscures the part of the specular reflectivity from which it would be possible to determine the Gaussian penetration depth $\sigma$. In analyzing data such as those in Fig. 9 for different temperatures, we therefore estimated $\sigma$ from Fig. 8 and used the fact that the exponential penetration length is equal to $\xi_{\|}(T)$, which is available from the published literature. Nonlinear least-squares fits were then carried out for the data in the vicinity of the peak, with the only free parameter being $B_{s}$. Best-fit values of $B_{s}$ versus temperature are listed in Table IV and plotted in Fig. 10 along with the high-resolution results from Table III. The solid line is the best power-law fit to the displayed data: $B_{s}=0.81\left(T-T_{N A}\right)^{(0.28 \pm 0.03)}$.

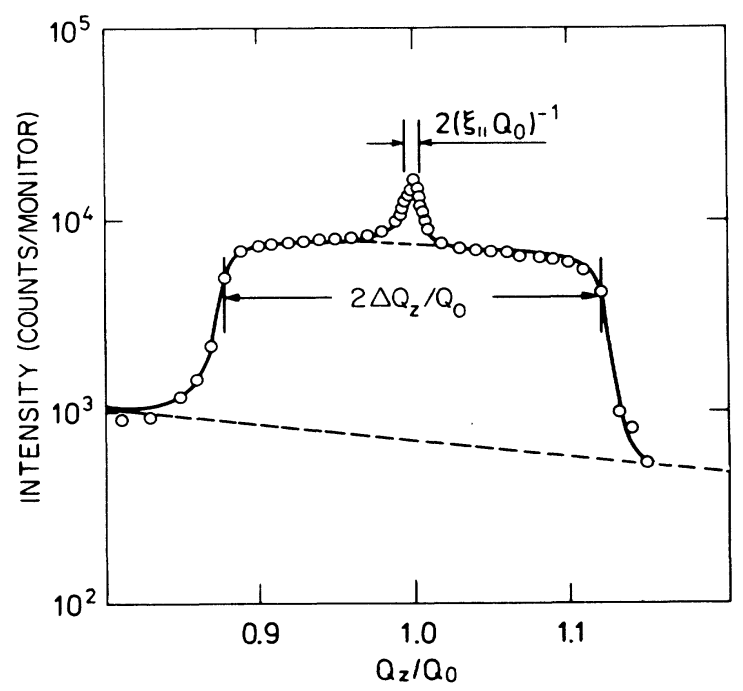

FIG. 9. A longitudinal scan (i.e., a $Q_{z}$ scan) from 8OCB at $T-T_{N A}=0.164^{\circ} \mathrm{C}$ with the low-resolution spectrometer illustrated in Fig. 1(c). The lower dashed line is the calculated Fresnel reflection law normalized to the intensity of $Q_{z} / Q_{0}=0.8$. The expected widths $\xi_{\|}$and $\Delta Q_{z}$ are indicated. 
TABLE IV. The results of nonlinear least-squares fits to low-resolution data for 8OCB. The correlation lengths $\xi_{\|}$and $\xi_{\perp}$ were taken from Ref. 18 for bulk 8OCB. Since the critical scattering form the bulk masks the falloff in $\Phi_{1}(Q)$ near $Q \simeq Q_{0}$, the fit is insensitive to the value of the Gaussian penetration length. Approximate values for $Q_{0} / \sigma$ are taken from Fig. 8 .

\begin{tabular}{ccccccc}
\hline \hline$T-T_{N A}$ & $\xi_{\|} Q_{0}$ & $\xi_{1} Q_{0}$ & $\sigma_{0} Q_{0}^{3}$ & $A^{\mathrm{a}}$ & $Q_{0} / \sigma$ & $B_{s}$ \\
\hline 0.011 & 1746 & 142 & $1.07 \times 10^{4}$ & $0.174 \times 10^{-2}$ & $10^{3}$ & $0.022 \pm 0.001$ \\
0.017 & 1279 & 110 & $1.01 \times 10^{4}$ & $0.182 \times 10^{-2}$ & $10^{3}$ & $0.024 \pm 0.001$ \\
0.022 & 1064 & 94.8 & $0.75 \times 10^{4}$ & $0.188 \times 10^{-2}$ & $10^{3}$ & $0.026 \pm 0.002$ \\
0.028 & 895 & 82.5 & $0.55 \times 10^{4}$ & $0.189 \times 10^{-2}$ & $10^{3}$ & $0.028 \pm 0.002$ \\
0.034 & 780 & 73.7 & $0.43 \times 10^{4}$ & $0.189 \times 10^{-2}$ & $10^{3}$ & $0.031 \pm 0.002$ \\
0.044 & 648 & 64.6 & $0.31 \times 10^{4}$ & $0.188 \times 10^{-2}$ & $10^{3}$ & $0.032 \pm 0.001$ \\
0.052 & 575 & 58.0 & $0.26 \times 10^{4}$ & $0.197 \times 10^{-2}$ & $10^{3}$ & $0.033 \pm 0.001$ \\
0.088 & 395 & 42.5 & $1.32 \times 10^{3}$ & $0.199 \times 10^{-2}$ & 110 & $0.039 \pm 0.002$ \\
0.164 & 253 & 29.6 & $0.60 \times 10^{3}$ & $0.203 \times 10^{-2}$ & 50 & $0.046 \pm 0.002$ \\
0.284 & 171 & 21.6 & $2.97 \times 10^{2}$ & $0.204 \times 10^{-2}$ & 20.0 & $0.054 \pm 0.002$ \\
0.553 & 106 & 14.6 & $1.24 \times 10^{2}$ & $0.203 \times 10^{-2}$ & 14.3 & $0.063 \pm 0.003$ \\
1.105 & 64.7 & 9.8 & $0.51 \times 10^{2}$ & $0.204 \times 10^{-2}$ & 7.7 & $0.073 \pm 0.002$ \\
\hline \hline
\end{tabular}

${ }^{\mathrm{a}} A=\sigma_{0} Q_{0}^{3} /\left[4 \pi^{2}\left(Q_{0} \xi_{\|}\right)\left(Q_{0} \xi_{1}\right)\right]$.

It is also evident from the data of Fig. 9 that the bulk critical scattering relative to the Fresnel intensity determines the absolute value of the amplitude of the critical smectic fluctuations. It is essentially only a matter of determining the effective volume $V$ contributing to bulk critical scattering. A ray incident at angle $\phi_{i}$ and specularly reflected at a depth $z$ below the surface is attenuated due to absorption by the amount $\exp \left(-2 z / l \sin \phi_{i}\right)$, where $l$ is the absorption length. By integration over $z$, the effective depth is therefore $(l / 2) \sin \phi_{i}$. The irradiated area is the cross-sectional beam area $A$ divided by $\sin \phi_{i}$. The effective scattering volume is therefore simply $V=A l / 2$.

The differential cross section $d \sigma / d \Omega$ due to density fluctuations $\delta \rho(\mathbf{r})$ in the volume $V$ of the electron density around its average value $\rho_{\mathrm{av}}$ is

$$
\begin{aligned}
\frac{d \sigma}{d \Omega} & =r_{0}^{2} V \int\langle\delta \rho(\mathbf{r}) \delta \rho(0)\rangle \exp (i \mathbf{Q} \cdot \mathbf{r}) d^{3} r \\
& \equiv r_{0}^{2} V \rho_{\mathrm{av}}^{2} S(\mathbf{Q})
\end{aligned}
$$

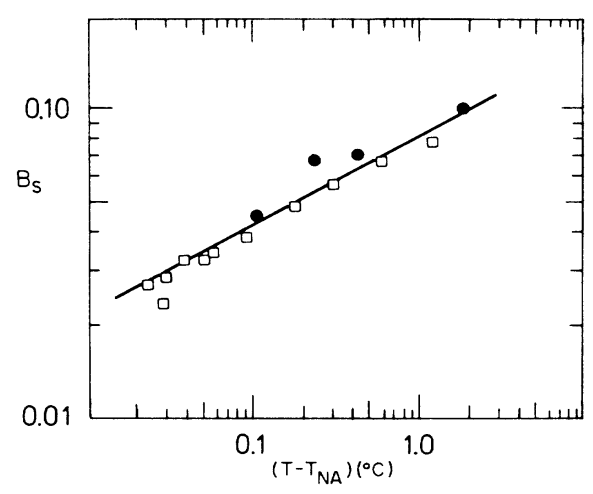

FIG. 10. The results obtained for $B_{s}$ from fits of the highresolution data shown in Fig. 6 (solid circles) and low-resolution data like those shown in Fig. 9 (open squares). The solid line represents $B_{s}=0.81\left(T-T_{N A}\right)^{0.28}$. where the last equation defines the scattering function $S(\mathbf{Q})$.

When the spectrometer is set for the central ray being incident at $\phi_{i}^{0}$ and scattered to angle $\phi_{s}^{0}$ in plane and $\psi_{s}^{0}$ out of plane, the recorded intensity $I_{d}$ is the folding of the cross section with the resolution function $R\left(\phi_{i}-\phi_{i}^{0}, \phi_{s}-\phi_{s}^{0}, \psi_{s}-\psi_{s}^{0}\right)$ :

$$
\begin{aligned}
I_{d}\left(\phi_{i}^{0}, \phi_{s}^{0}, \psi_{s}^{0}\right) & \\
=I_{0} \iiint & \frac{d \sigma}{d \Omega}\left(\phi_{i}, \phi_{s}, \psi_{s}\right) R\left(\phi_{i}-\phi_{i}^{0}, \phi_{s}-\phi_{s}^{0}, \psi_{s}-\psi_{s}^{0}\right) \\
& \times d \phi_{i} d \phi_{s} d \psi_{s} .
\end{aligned}
$$

Since the cross section is expressed in terms of wave vector $\mathbf{Q}$ rather than $\left(\phi_{i}, \phi_{s}, \psi_{s}\right)$ it is convenient to carry out the folding integral in $\mathbf{Q}$ space rather than angular space. The relation between the volume element in $\mathbf{Q}$ space and that in angular space can be inferred from the kinematics in Fig. 1,

$$
d^{3} Q=\left(k^{2} Q \cos \phi_{i}\right) d \phi_{i} d \phi_{s} d \psi_{s} .
$$

The parallelogram is an area element in $Q_{y}-Q_{z}$ space. Denoting the short side by $\mathbf{X}_{i}$ and the long side by $\mathbf{X}_{s}$ with lengths $k \delta \phi_{i}$ and $k \delta \phi_{s}$, respectively, we find the area to be

$$
\begin{aligned}
k^{2} \delta \phi_{i} \delta \phi_{s} \sin (2 \phi) & =2(k \sin \phi)(k \cos \phi) \delta \phi_{i} \delta \phi_{s} \\
& =Q k(\cos \phi) \delta \phi_{i} \delta \phi_{s} .
\end{aligned}
$$

The out-of-plane component is of course $k \delta \psi_{s}$ and thereby the volume element is as given by Eq. (20).

Finally, from the definition of $\phi_{c}^{2}=\lambda^{2}\left(\rho_{\mathrm{av}}\right) r_{0} / \pi$, using Eq. (1a),

$$
\left(\rho_{\mathrm{av}} r_{0}\right)^{2}=\left(\frac{Q^{4}}{16 \pi^{2}}\right) R_{F}(Q),
$$

and on substitution into Eq. (18), one obtains the following expression for the bulk intensity at $Q^{0}$ : 


$$
\begin{aligned}
I_{\text {bulk }}\left(\mathbf{Q}^{0}\right)= & I_{\text {Fresnel }}\left(Q_{z}\right)\left(\frac{Q^{4}}{16 \pi^{2}}\right)\left(\frac{l}{2}\right)\left(k^{2} Q \cos \phi\right)^{-1} \\
& \times \int g\left(\mathbf{Q}-\mathbf{Q}^{\prime}\right) S\left(\mathbf{Q}^{\prime}\right) d^{3} Q^{\prime},
\end{aligned}
$$

where $g\left(\mathbf{Q}-\mathbf{Q}^{\prime}\right)$ is the resolution function in reciprocal space. For $S(\mathrm{Q})$ we take the form used in Ref. 18,

$$
S(\mathbf{Q})=\sigma_{0}\left[1+\xi_{\|}^{2}\left(Q_{z}-Q_{0}\right)^{2}+\xi_{\perp}^{2} Q_{\perp}^{2}\left(1+c \xi_{\perp}^{2} Q_{\perp}^{2}\right)\right]^{-1},
$$

with the values of $\xi_{\|}$and $\xi_{\perp}$ and $c$ versus temperature as determined in Ref. 18. The only unknown parameter in Eqs. (22) and (23) is therefore $\sigma_{0}$, which has the dimension of volume. A least-squares fit to the data obtains the values of $\sigma_{0} Q_{0}^{3}$ given in Table IV and plotted in Fig. 11.

The physical significance of $\sigma_{0}$ is better appreciated upon taking the Fourier transform of $S(\mathbf{Q})$. We define the dimensionless distance $s$ by

$$
s^{2} \equiv\left[x^{2}+y^{2}+\left(\frac{\xi_{\perp} z}{\xi_{\|}}\right)^{2}\right]\left(\frac{Q_{0}}{2 \pi}\right)^{2} .
$$

In $s$ space, where the critical fluctuations are isotropic, one obtains the correlation function

$$
\begin{aligned}
& \rho_{\mathrm{av}}^{-2}\langle\delta \rho(\mathbf{r}) \delta \rho(0)\rangle \\
& \quad \simeq\left(\frac{\sigma_{0} Q_{0}^{3}}{4 \pi^{2}\left(Q_{0} \xi_{\|}\right)\left(Q_{0} \xi_{\perp}\right)}\right) s^{-1} \exp \left(\frac{-2 \pi s}{Q_{0} \xi_{1}}\right) \cos \left(Q_{0} z\right) .
\end{aligned}
$$

The quantity in square brackets is a measure of the absolute value of the density-density correlation function. It is also listed in Table IV. It is essentially independent of temperature with an average value around 0.0020 .

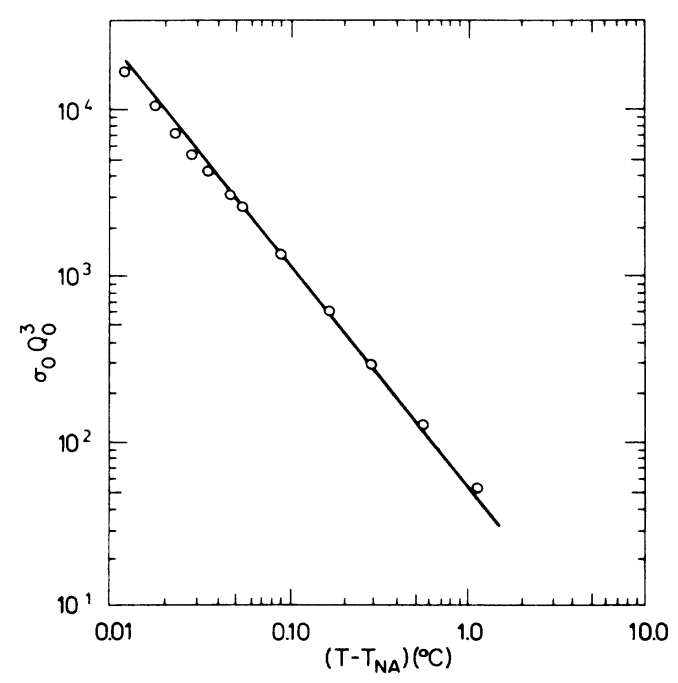

FIG. 11. The amplitude $\sigma_{0} Q_{0}^{3}$ of the critical scattering from the bulk of $8 O C B$ as a function of $T-T_{N A}$. The solid line has the slope -1.32 that was previously measured in a transmission geometry (Ref. 18).

\section{8OCB, transverse scans, wavelength dependence}

So far we have only shown longitudinal scans to support the model of surface smectic layering accompanied by critical scattering from the bulk. Figure 12 displays transverse out-of-plane scans at two different wavelengths. The spectrometer parameters are listed in Table II under entry 3.83. The height of slit $S_{3}$ is maintained relatively open at $4 \mathrm{~mm}$; however, its width is reduced to $0.2 \mathrm{~mm}$. As illustrated in Figs. 12(e) and 12(f), the $Q_{z}-Q_{y}$ cross section of the resolution volume is still approximated by a long thin parallelogram, as in the case of Fig. 1(c). The scans in Fig. 12 are in the $Q_{x}$ direction, or normal to the $Q_{z}-Q_{y}$ plane, at fixed values of $Q_{z} / Q_{0}=1.0$ [Figs. 12 (a) and $12(\mathrm{c})$ ] and $Q_{z} / Q_{0}=1.04$ [Figs. 12(b) and 12(d)]. The latter choice is motivated by looking at Fig. 5-here the wave scattered from the surface layering and the ordinary Fresnel wave interfere destructively and the total surface scattering, confined to the $Q_{z}$ axis, is therefore very

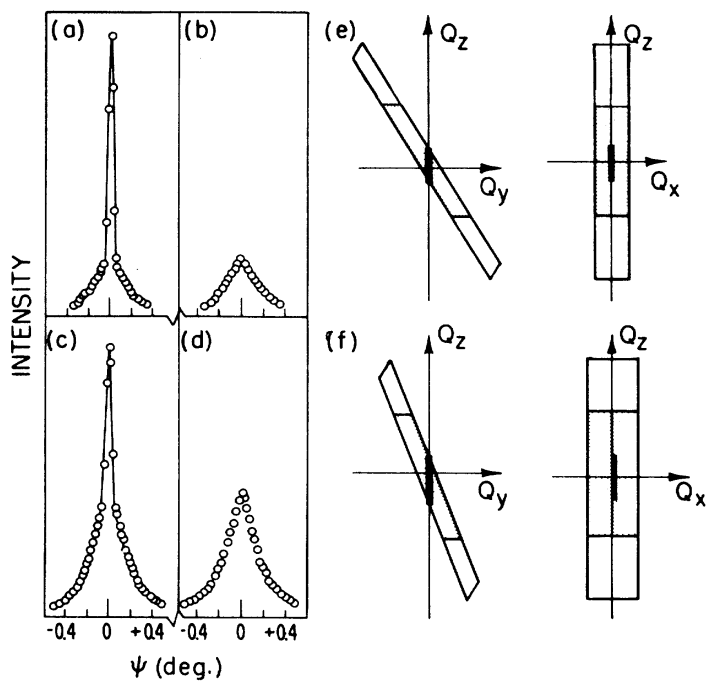

FIG. 12. Transverse out-of-plane scan along $Q_{x}$ as a function of the angle $\psi \approx Q_{x} /\left[k \cos \left(\phi_{i}\right)\right]$ for $8 \mathrm{OCB}$ at $T-T_{N A}$ $=0.585^{\circ} \mathrm{C}$. The $\mathrm{x}$-ray wavelength for (a) and (b) is $\lambda=1.543 \AA$ and for (c) and (d) $\lambda=0.943 \AA$. For (a) and (c) the spectrometer is set such that the resolution volume is centered at $Q_{y}=0$ and $Q_{z} / Q_{0}=1$ [see Fig. 1(c)] and the scan shows both the critical scattering from the bulk and the surface peak. For (b) and (d) $Q_{y}=0$ and $Q_{z} / Q_{0}=1.04$. From the data in Fig. 5 one can see that the specular reflection is nearly zero and these scans are essentially only the bulk critical scattering. The signals from the bulk at $Q_{z} / Q_{0}=1.0$ and at $Q_{z} / Q_{0}=1.04$ are equal; however, the ratio of specular to bulk varies with wavelength. The intersections between $Q_{z}-Q_{y}$ and $Q_{z}-Q_{x}$ cross sections of the resolution parallelogram and the scattering are shown for $\lambda=1.543$ $\AA$ (e) and $\lambda=0.943 \AA$ (f). The heavy line indicates the specular reflectivity and the crosshatched region indicates the bulk scattering. As discussed in the text the ratio of specular reflection to bulk would be independent of wavelength were it not for irradiated volume effects. 
weak and the scattering even at $Q_{x} \simeq k \psi=0$ is therefore due to bulk critical fluctuations. Data such as those in Fig. 12(b) are therefore particularly useful to obtain the transverse correlation range $\xi_{1}$. A least-squares fit to the cross section given in Eq. (23), properly folded with the resolution function, gives $\xi_{\perp}$ versus temperature as shown in Fig. 13 compared to previous bulk data (solid line) from Ref. 18. The agreement is excellent.

We shall now discuss the wavelength dependence of surface scattering relative to bulk scattering. The surface scattering is indicated by the solid line in Figs. 12(a) and 12(c). The data in Figs. 12(b) and 12(d) provide a very accurate determination of the bulk intensity so that the separation into surface and bulk contribution at $Q_{z} / Q_{0}=1.0$ does not depend on an analysis of two superimposed line shapes. The conditions for the data for $\lambda=0.943 \AA$ in Figs. 12(c) and 12(d) are identical to those for $\lambda=1.543 \AA$ in Figs. 12(a) and 12(b), except that we here have used another set of monochromator planes, $\mathrm{Si}(220)$, and have changed the tilt accordingly.

The bulk scattering relative to the surface scattering is considerably enhanced at the small wavelength, by a factor of about 3.3. The reason is of course that the smaller wavelength radiation penetrates deeper into the bulk and therefore picks up more effective volume.

For a quantitative discussion two effects must be considered. The first is the wavelength dependence of the effective volume for bulk scattering, in relation to the effective area for specular reflection. We have argued above that the effective volume for bulk scattering is the crosssectional beam area $A$ times half of the absorption length $l(\lambda)$. The effective area for surface scattering is $A / \phi_{i} \sim A / \lambda$. Thus the ratio of bulk scattering to surface scattering should vary as $\lambda l(\lambda)$. A second point to consider, but which proves to be unimportant, is the $\lambda$ dependence of the effective overlap of the resolution function with the specular reflection and with the bulk critical scattering. This is illustrated in Figs. 12(e) and 12(f) for the identical slit configurations used at $\lambda=1.543$ and

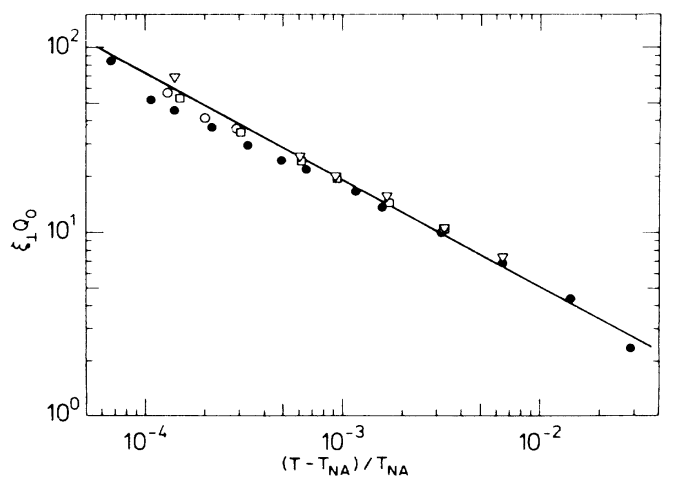

FIG. 13. Results of nonlinear least-squares fits for the transverse bulk correlation length $\xi_{\perp}$ vs $T-T_{N A}$ for $80 \mathrm{OB}$ from data like those in Fig. 12 for $\lambda=0.77 \AA$ (circles), $\lambda=0.94 \AA$ (squares), and $\lambda=1.54 \AA$ (triangles). The solid line is the previously published result for $\xi_{\perp}$ (Ref. 18).
$0.943 \AA$. The shaded areas indicating bulk scattering overlap in the $Q_{y}-Q_{z}$ plane are independent of $\lambda$, but in the $Q_{x}-Q_{z}$ plane, the resolution width varies as $k$ or $1 / \lambda$. Altogether the bulk scattering overlap varies as $1 / \lambda$. However, the surface scattering overlap, indicated by the heavy bar also varies as $1 / \lambda$ because the resolution parallelogram is steeper at shorter wavelengths. We conclude that there is no $\lambda$ dependence due to resolution effects in the ratio of bulk-to-surface signal. The measured ratio of bulk-to-surface signal is expected to vary as $\lambda l(\lambda)$ or as $\lambda^{-2}$ since the measured $l(\lambda)$ varies approximately as $\lambda^{-3} \cdot{ }^{27}$ For the wavelengths in Fig. 12 the ratio is $(0.943 / 1.543)^{-2}=2.7$, to be compared with the experimental value of 3.3. In view of the experimental uncertainties, we believe this agreement is reasonable.

In concluding this section we have demonstrated by transverse scans with appropriate slit widths that the bulk scattering is due to critical smectic fluctuations in the bulk with a finite width $\left(\xi_{1} Q_{0}\right)$ in contrast to the surface smectic layering which as essentially the infinite correlation of the surface. This interpretation has been confirmed by the wavelength dependence of the bulk signal relative to the surface signal.

\section{40.8 intermediate resolution}

The geometries used for the study of $80 C B$ were rather extreme: very high resolution obtained by narrow slit heights and a channel-cut, perfect analyzer crystal, or low resolution obtained by a slit aperture in $S_{3}$ which was several millimeters either in one or both directions. The entire study of both bulk and surface properties throughout the nematic region can be carried out at an intermediate resolution with no analyzer crystal but with $S_{3}$ slit dimensions of a few tenths of millimeters in both directions.

The most important consequence of this is that the $Q_{y}-Q_{z}$ projection of the resolution function is changed from the long thin parallelogram of Fig. 1, tilted at angle $\phi_{i}$ to the $Q_{z}$ axis, to a more symmetric figure with the symmetry axis approximately parallel to the $Q_{z}$ axis.

The material butyloxybenzylidene octylanine (4O.8) was studied using this configuration. The principle difference between 40.8 and $80 C B$ is that in 40.8 the smectic layer spacing $d$ is essentially equal to the length of the molecule, while for $8 \mathrm{OCB}$ the molecules overlap each other and the layer spacing is approximately 1.6 times the length of the individual molecules. ${ }^{20}$ Data shown in Fig. 14 are for a temperature $0.5^{\circ} \mathrm{C}$ above the nematic-tosmectic- $A$ transition temperature. The filled circles in Fig. 14(a) are a longitudinal scan on the $Q_{z}$ axis with $Q_{x}=Q_{y}=0$. The open circles below are a similar scan but with the out-of-plane wave vector misset, $Q_{x} / Q_{0}=0.05$. The later data represent the longitudinal bulk scattering. Notice the logarithmic intensity scale. The two peaks appear to have approximately the same widths: a direct demonstration that the penetration depth of surface smectic layering equals the bulk correlation range. The solid lines are least-squares fits of the model described in the preceding sections. The data for $Q_{x} / Q_{0}=0.05$ determine the bulk correlation range $\xi_{||} Q_{0}=93$ and the $\sigma_{0} Q_{0}^{3}=256$. 


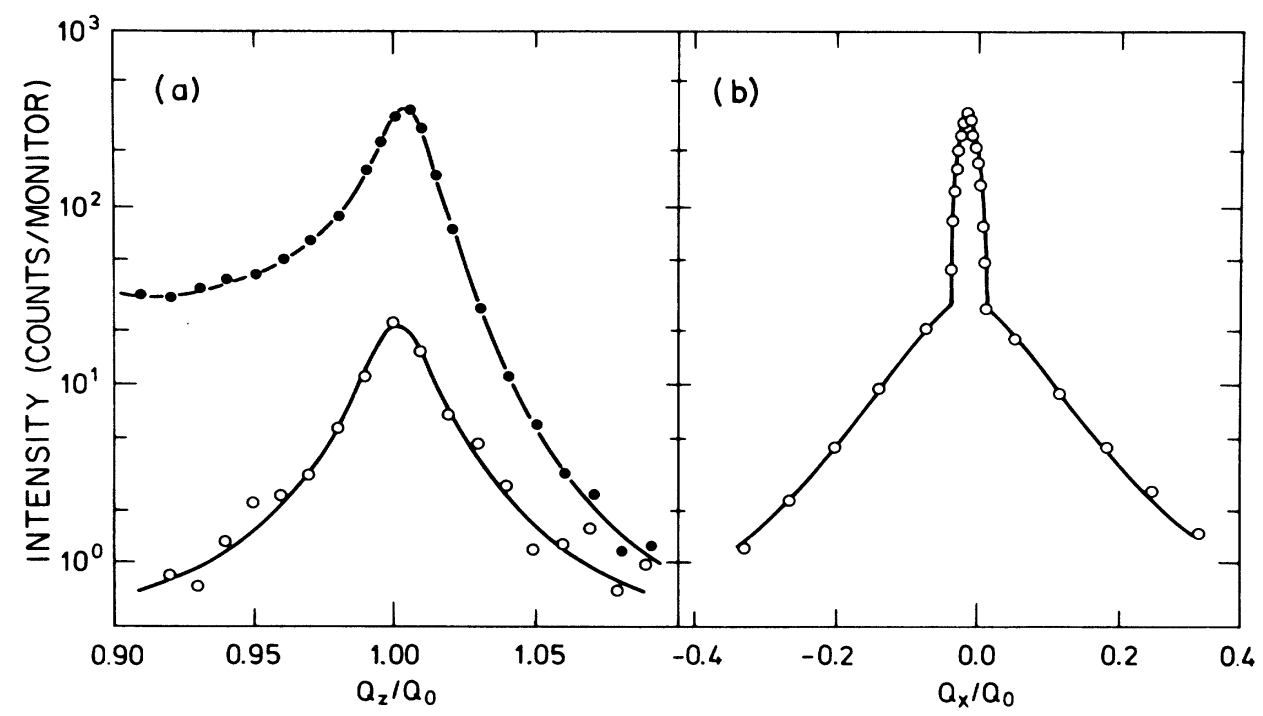

FIG. 14. Intermediate-resolution data for 40.8 at $T-T_{N A}=0.5^{\circ} \mathrm{C}$. (a) $Q_{z}$ scans at $Q_{x}=0$ (filled circles), corresponding to the sum of the specular reflection and the bulk critical scattering and at $Q_{x} / Q_{0}=0.05$ (open circles), which is only the bulk signal. (b) $Q_{x}$ scans for $Q_{z} / Q_{0}=1.0$ in which the specular and bulk are clearly separable.

The data for $Q_{x} / Q_{0}=0$ determine the penetration depth $\xi_{\|} Q_{0}=99$, the surface amplitude $B_{s}=0.095$, and the Gaussian depth $Q_{0} / \sigma=7 \pm 3$, assuming the phase $z_{0}=0.25 d$ as for 8OCB. The quality of both fits is very good. The data in Fig. 14(b) are a transverse out-of-plane scan similar to those of Fig. 12 for 8 OCB. The bulk and surface scattering are clearly separated into a broad and a narrow, resolution-limited peak. These data then determine the transverse bulk correlation range $\xi_{\perp} Q_{0}=11.2$.

The three types of scans displayed in Fig. 14 contain all information on the free surface of a liquid crystal in the nematic phase. The reflected waves from the surface and the smectic surface layering interfere giving rise to an asymmetric line shape for the total surface scattering. Its width determines the penetration depth of surface layering, its height determines amplitude of the surfaceinduced smectic order. The bulk scattering is dominated by the critical scattering from smectic- $A$ fluctuations in the nematic phase characterized by the longitudinal and transverse correlation ranges $\xi_{\|} Q_{0}$ and $\xi_{1} Q_{0}$, respectively, as well as the absolute magnitude of the density correlation function as expressed by the parameter $\sigma_{0} Q_{0}^{3}$. Data sets such as those in Fig. 14 were taken at a number of temperatures and the best-fit values of model parameters discussed above are listed in Table $\mathrm{V}$ and displayed in Fig. 15. The dashed lines are the results previously obtained for the critical scattering from the bulk of 40.8 in a transmission geometry, ${ }^{19}$ except that in that experiment the absolute value of $\sigma_{0} Q_{0}^{3}$ was not measured. Best fits to the displayed data obtain $\sigma_{0} Q_{0}^{3}=130\left(T-T_{N A}\right)^{-1.26 \pm 0.05}$. As with $8 \mathrm{OCB}$, measurements of the bulk properties in the two different geometries agree. The Gaussian penetration length and the amplitude $B_{s}$ are also similar to those of 8OCB and the best fits obtain $Q_{0} / \sigma=5.0(T$ $\left.-T_{N A}\right)^{-0.51 \pm 0.05}$ and $B_{s}=0.10\left(T-T_{N A}\right)^{+0.10 \pm 0.05}$. Although the critical exponents $\delta$ and $v_{\|}$and $v_{\perp}$ are essen- tially equal for $8 \mathrm{OCB}$ and 40.8 , the "exponents" for $B_{s}$ are significantly different.

\section{DISCUSSION}

The principle purpose of this manuscript is to present experimental results demonstrating that the $\mathrm{x}$-ray reflectivity of nematic liquid-crystal surfaces display features indicative of surface-induced smectic order. These features are unambiguously distinguished from all other scattering processes by the existence of a two-dimensional selection rule, e.g., $Q_{x}=Q_{y}=0$. The most striking property of this order is the fact that it penetrates into the bulk exponentially with a characteristic length that is equal to the parallel correlation length $\xi_{\|}$, for critical fluctuations. In addition to the sharp peak, the $\mathrm{x}$-ray reflectivity displays characteristic deviations from the Fresnel reflection law. We demonstrated above that the total reflectivity could be modeled by $R(\phi)=R_{F}(\phi)|\Phi|^{2}$, where $\Phi=\Phi_{0}+\Phi_{1}$. The first of these $\Phi_{0}$, the Fourier transform

TABLE V. The results of nonlinear least-squares fits for the intermediate-resolution data from 40.8 .

\begin{tabular}{crcccc}
\hline $\begin{array}{c}T-T_{N A} \\
\left({ }^{\circ} \mathrm{C}\right)\end{array}$ & $\sigma_{0} Q_{0}^{3}$ & $\xi_{\|} Q_{0}$ & $\xi_{1} Q_{0}$ & $Q_{0} / \sigma$ & $B_{s}$ \\
\hline 0.019 & 25870 & 933 & 113 & $\mathrm{a}$ & 0.056 \\
0.094 & 3640 & 332 & 46 & 12.45 & 0.076 \\
0.134 & 1554 & 233 & 30 & 8.6 & 0.082 \\
0.254 & 682 & 145 & 21.1 & 7.67 & 0.088 \\
0.510 & 284 & 91.8 & 13.6 & 6.27 & 0.095 \\
2.006 & 45 & 31.7 & 5.06 & 3.56 & 0.112 \\
\hline \hline
\end{tabular}

${ }^{\text {a }}$ Could not be determined because of high uncertainty. 


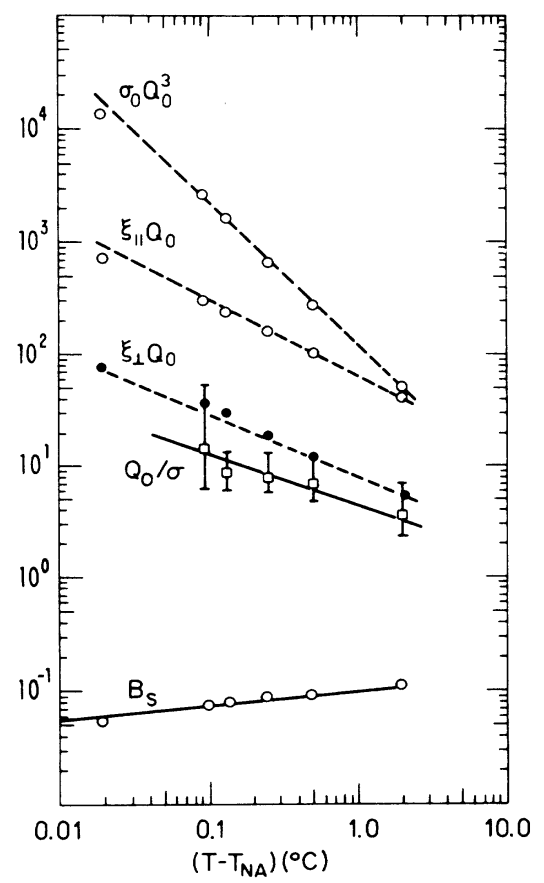

FIG. 15. Temperature dependence of the parameters obtained from nonlinear least-squares fits of the intermediateresolution data for 40.8 as listed in Table V. The dashed lines through the data represent previously published results (Ref. 19) for the amplitude and slope, $\xi_{\|}$and $\xi_{1}$, and for the slope of $\sigma_{0} Q_{0}^{3}$. The solid lines are fits to the data as discussed in the text.

of the exponentially penetrating surface oscillations, is theoretically motivated and the only adjustable parameters that were not previously known are the amplitude of the oscillations, $B_{s}$, and the phase factor, $z_{0}$, that defines the phase of the oscillations relative to the surface.

The second, or $\Phi_{1}$, term was represented by an ad hoc form that was simply taken because it was capable of representing the data. On the other hand, the functional form of $\Phi_{1}$ has features that are essential to any full theoretical representation of the surface layers. For example, from Figs. 8 and 15 one can see that the Gaussian penetration length $Q_{0} / \sigma$ of $\Phi_{1}$ is at least two to three times smaller than the exponential penetration length $\xi_{\|}$ of $\Phi_{0}$. The implication is that although the simple exponential decay of the $\Phi_{0}$ term does describe the main peak in the reflectivity it is not adequate to describe the amplitude of the real-space smectic oscillations near the surface. We believe that the counterintuitive temperature dependence in which $B_{s}$ approaches zero as $T$ approaches $T_{N A}$ is a reflection of the interplay between these two terms. One consequence of this is that the amplitude of the smectic order parameter near to the surface is not just equal to $B_{s}$ but is actually the amplitude of the real-space oscillations that are represented here by the sum of the $\Phi_{0}$ and $\Phi_{1}$ terms.

An alternative approach that has recently been applied to the interpretation of antiferroelectric layering at the surface of a different type of liquid-crystal material assumes that the surface fixes the position and orientation of the surface molecules and then calculates the electron density profile near the surface from models of the molecular form factor. ${ }^{28}$ For both 8OCB (Ref. 29) and 40.8, such a calculation obtains amplitudes for the Fourier component of the electron density at $Q_{0}$ of the order of 0.1 . It is interesting to note that the amplitudes for the real-space electron density oscillations associated with the $\Phi_{1}$ term are of the same order of magnitude. Since in 8OCB, for $T \approx T_{N A}, B_{s}$ is considerably smaller than 0.1 , the current model for $\Phi_{1}$ is in reasonable agreement with the molecular model near to $T_{N A}$. For $80 \mathrm{OB}$, at higher temperatures, $B_{s}$ approaches 0.1 and the predictions from the molecular model must be compared to the density obtained from the sum of the $\Phi_{0}$ and $\Phi_{1}$ terms. Since the reasonable agreement that can be obtained is very sensitive to the poorly determined phase $z_{0}$, detailed comparisons are not warranted. A similar situation prevails for $4 \mathrm{O} .8$ over the entire measured temperature range. Nevertheless, the chosen phase factor, $z_{0} \approx 0.25 d$, can be justified by inspection of the molecular models.

Finally, we have made a direct comparison between the diffuse critical scattering from the bulk and the specular reflection from the surface. Since the latter follows the Fresnel reflection law whose value is known absolutely in terms of the Thompson cross section of the electron, this obtains a measure of the absolute value of the critical cross section. We have interpreted this to obtain an absolute value for the critical part of the density-density correlations.

\section{ACKNOWLEDGMENTS}

This work was supported in part by grants from the Danish National Science Foundation, by the Ris $\varnothing$ National Laboratory, by the U.S. National Science Foundation through Grant Nos. DMR-82-12189, DMR-83-16979, and INT-83-11841, and by the U.S. Joint Services Electronics Program (U.S. Army, Navy, and Air Force) through Grant No. N00014-84-K-0465.
*Permanent address: Department of Physics, University of Texas at Arlington, P.O. Box 19059, Arlington, TX 76019.

${ }^{1}$ H. Kiessig, Ann. Phys. 10, 769 (1931).

${ }^{2}$ A. H. Compton and S. K. Allison, X-Rays in Theory and Experimentation (Van Nostrand, New York, 1935).

${ }^{3}$ L. G. Parratt, Phys. Rev. 95, 359 (1954).
${ }^{4}$ L. Nevet and P. Croce, Rev. Phys. Appl. 15, 761 (1980).

${ }^{5}$ D. H. Bilderback and S. Hubbard, Nucl. Instrum. Methods 195, 85 (1982); 195, 91 (1982).

6J. A. Prins, Z. Phys. 47, 479 (1928).

${ }^{7}$ E. Nahrig, Phys. Z. 31, 401 (1930).

${ }^{8}$ L. A. Smirnov, Opt. Spektrosk. 43, 567 (1977) [Opt. Spectrosc. 
(USSR) 43, 333 (1977)].

${ }^{9}$ Synchrotron Radiation Research, edited by H. Winick and S. Doniach (Plenum, New York, 1980).

${ }^{10}$ Synchrotron Radiation, Techniques and Applications, edited by C. Kunz (Springer-Verlag, Berlin, 1979).

${ }^{11}$ I. M. Bloch, M. Sansone, F. Rondelez, D. G. Peiffer, P. Pincus, M. W. Kim, and P. M. Eisenberger, Phys. Rev. Lett. 54, 1039 (1985).

12J. Als-Nielsen, F. Christensen, and P. S. Pershan, Phys. Rev. Lett. 48, 1107 (1982).

13J. Als-Nielsen and P. S. Pershan, Nucl. Instrum. Methods 208, 545 (1983).

14P. S. Pershan and J. Als-Nielsen, Phys. Rev. Lett. 52, 759 (1984).

${ }^{15}$ A. Braslau, M. Deutsch, P. S. Pershan, A. H. Weiss, J. AlsNielsen, and J. Bohr, Phys. Rev. Lett. 54, 114 (1985); A. Braslau, B. M. Ocko, P. S. Pershan, J. Als-Nielsen, and M. Deutsch (unpublished).

${ }^{16}$ M. P. D'Evelyn and S. A. Rice, Phys. Rev. Lett. 47, 1844 (1981); D. S. Sluis, M. P. D'Evelyn, and S. A. Rice, J. Chem. Phys. 78, 545 (1983).

${ }^{17}$ T. Lubensky, J. Chem. Phys. 80, 31 (1983).

${ }^{18}$ J. D. Litster, J. Als-Nielsen, R. J. Birgeneau, S. S. Dana, D.
Davidor, F. Gareia-Golding, M. Kaplan, C. R. Safinya, and R. Schaetzing, J. Phys. (Paris) Colloq. 40, C3-339 (1979).

${ }^{19}$ R. J. Birgeneau, C. W. Garland, G. B. Kasting, and B. M. Ocko, Phys. Rev. A 24, 2624 (1981).

${ }^{20}$ A. J. Leadbetter, C. Frost, J. P. Gaughan, G. W. Gray, and A. Mosley, J. Phys. (Paris) 40, 375 (1979).

${ }^{21}$ B. B. Warren, $X$-Ray Diffraction (Addison-Wesley, Reading, MA, 1969).

${ }^{22}$ M. Nielsen and H. B. Møller, Acta Cryst. A 25, 547 (1969); B. Lebech and M. Nielsen, Proceedings of the Neutron Diffraction Conference, Petten, The Netherlands, RCN-234 (1975), p. 466.

${ }^{23}$ V. Bonse and M. Hart, Appl. Phys. Lett. 7, 238 (1965).

${ }^{24}$ F. J. Kahn, Appl. Phys. Lett. 22, 386 (1973).

${ }^{25}$ B. K. Agarwal, X-Ray Spectroscopy (Springer-Verlag, Berlin, 1979), p. 178.

${ }^{26}$ B. Ocko, A. Braslau, P. S. Pershan, J. Als-Nielsen, and M. Deutsch, Phys. Rev. Lett. 57, 94 (1986).

${ }^{27}$ International Tables for X-Ray Crystallography (Kynoch Press, Birmingham, England, 1962).

${ }^{28}$ E. F. Gramsbergen, W. H. de Jeu, and J. Als-Nielsen, J. Phys. (Paris) 47, 711 (1986)

${ }^{29}$ B. Ocko (private communication). 